
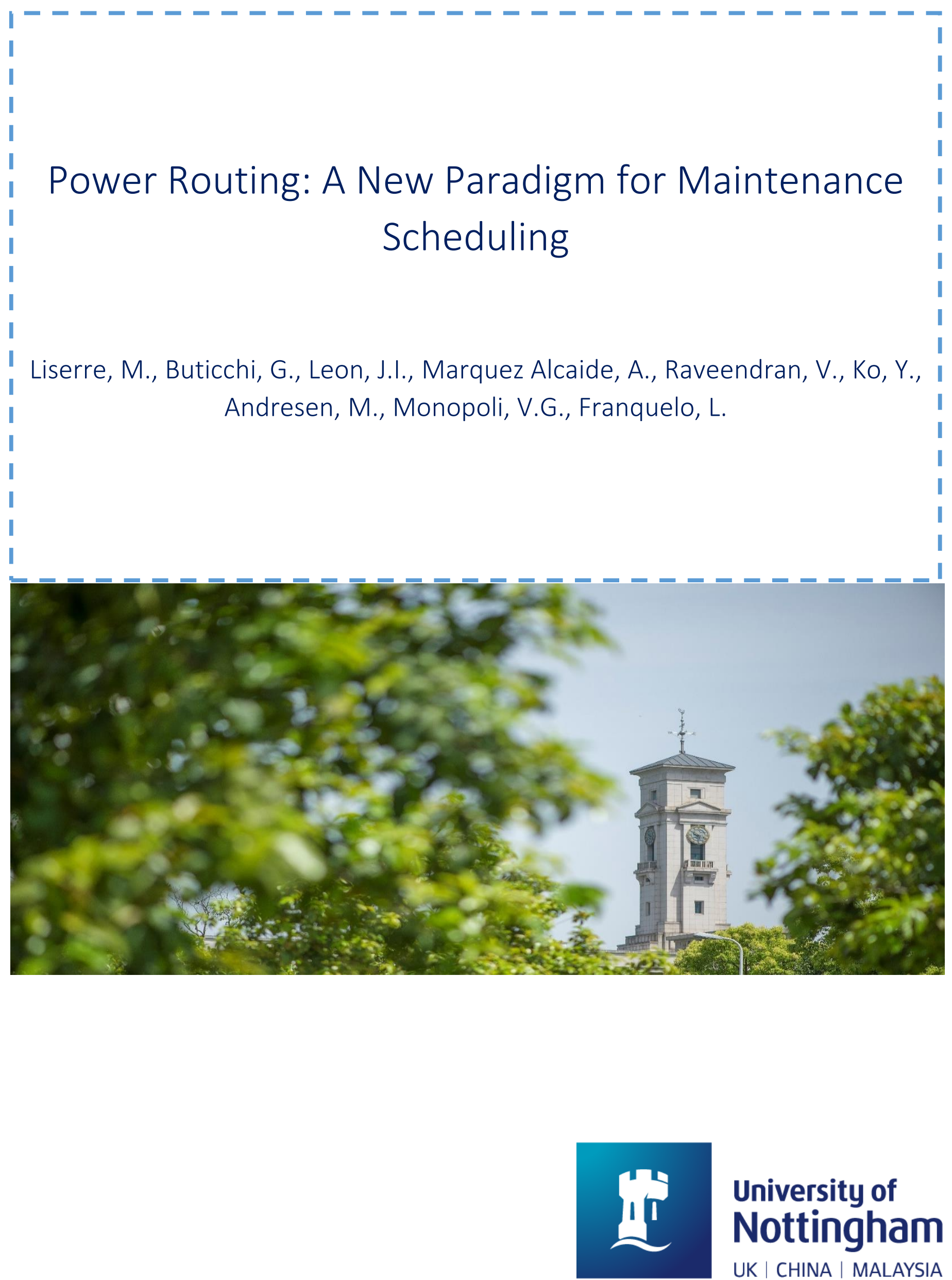

University of Nottingham 
University of Nottingham Ningbo China, 199 Taikang East Road, Ningbo, 315100, Zhejiang, China.

First published 2020

This work is made available under the terms of the Creative Commons Attribution 4.0 International License:

http://creativecommons.org/licenses/by/4.0

The work is licenced to the University of Nottingham Ningbo China under the Global University Publication Licence:

https://www.nottingham.edu.cn/en/library/documents/researchsupport/global-university-publications-licence-2.0.pdf 


\section{Power Routing: a New Paradigm for Maintenance Scheduling}

\section{INTRODUCTION}

Nowadays, the necessity of efficient and reliable power systems is increasing in order to meet the strict requirements imposed by standards and regulations with minimum cost. One specific requirement is focused on the maximization of the power converters lifetime what produces a reduction in the maintenance cost. However, to overcome the related challenges is not a straight-forward task as it involves knowledge of power devices physics, smart management of electrical magnitudes and optimal maintenance planning and scheduling. It represents a multidisciplinary issue being faced in the last decade. As a first issue to be considered, it is clear that the evolution of power electronics is playing a principal role in the world in many ways:

- development of environmentally friendly and economically affordable clean energy sources such as the wind and solar energy [1],

- installation and enhancement of facilities to develop the robust, reliable and high quality distributed electrical grid including the smart grid paradigm with distributed energy storage systems [2]-[5],

- efficient and low cost freight transportation based on electric vehicles, low-fuel consumption cargo ships and more electric aircraft [6],

- personal transportation such as electric motorbikes, electric cars, robust high-speed trains, efficient subways with regenerative breaking, public electric buses, etcetera [7].

The power electronic applications, that include multi-disciplinary research topics such as digital signal processing, power electronics and power systems, have actually experienced a technology disruption in the last decades [8]: 1) new power semiconductor devices with high voltage/current ratings and very low power losses; 2) powerful microprocessor hardware platforms including accurate sensors and high-bandwidth data acquisition systems; 3) effective simulation and design software tools to predict the behavior of power systems.

Power electronics reliability and consequently the corresponding required power systems maintenance cost is one of the major concerns of this technology [9]. An accurate estimation of the remaining useful lifetime (RUL) of a power converter is fundamental to reduce the maintenance cost [10]. Historically, the first approaches to estimate the RUL were just limited by the manufacturers guarantee. The power system operator just counted the power converter years in operation to decide when it had to be replaced by a new unit. But it is clear that the accuracy of this approach is low because the RUL highly depends on the operating conditions of the power system, usually called mission profile. For instance, factors such as the ambient temperature and the actual power that is managed by the power system highly influences the possibility of failure in any power converter component [11]. 
A more accurate RUL estimation presents several advantages [12]. Firstly, it allows for reduced safety margins, resulting in cheaper and often lighter systems. As a second advantage, it enables to reduce the cost of unscheduled maintenance. For example, in photovoltaic system $60 \%$ of these costs are caused by the inverter [21]. The design of an efficient maintenance schedule allows substituting the system components when their failure probability achieve a maximum limit. This feature is especially beneficial in mission-critical systems, such as aircraft transportation or power distribution, where a failure is critical due to safety reasons.

In addition to the maintenance scheduling with accurate RUL estimations, the power systems availability is usually enhanced by developing power converters with inherent fault tolerant capability [13]. It means that, even if a failure happens in the system, it can be still operational but in most of the cases with reduced nominal power. However, this feature is not easy to be achieved in many cases. In Fig. 1a), a power rectifier is presented consisting of a two-level active rectifier connected to a dual active bridge dc/dc converter (DAB). This power system does not own fault tolerant capability because if a component of the power converter fails, the whole system must stop its operation.

The fault tolerant capability feature can be achieved by designing power converter topologies with high modularity [14]. In this way, a modular power converter is formed by the serial or parallel connection of basic power modules, also called power electronic building blocks - PEBB. Modular power converters have inherent fault tolerant capability by bypassing the damaged PEBBs after a failure. PEBB-based power converters are a very attractive solution for medium- and high-power applications where a high voltage/current range can be achieved by the serial/parallel connection of low-voltage PEBB [15].

In addition, modular power converters generate high quality output waveforms reducing the required filtering stage and also present advantages from the point of view of the mass production of PEBBs [16]. However, the component count is higher which could still be seen in industry as a possible cause of more failures if old approaches on reliability prediction based on part count are considered. In any case, the system availability will be higher [17]. The knowledge of the RUL is particularly beneficial in order to maximize the reliability and to preserve the highest availability, which a modular system could guarantee [18].

An example of modular system is shown in Fig. 1b), consisting of a cascaded H-bridge converter (CHB) connected to multiple dc/dc quadruple active bridge converters (QAB). In this modular system, even if a failure happens in some PEBB, the system can be still in operation. For instance, in the case of the CHB, a bypass switch can be added to the H-bridge-based PEBB to overcome the failure in the corresponding PEBBs [19]. Fault diagnosis methods are applied to find out the damaged PEBBs carrying out the bypass and adapting the power converter control to achieve optimal operation [20]. A summary of advantages and drawbacks of modular converters is also included in Fig. 1.

From a cost reduction point of view, it is clear that it would be beneficial if the RUL of the converter devices could be adjusted according to the maintenance requirements. If this feature were achieved, the PEBBs could adapt their operation to fit with the scheduled maintenance, making the planned maintenance tasks more cost efficient.

This paper describes how the basic idea of managing the operation of the single PEBBs in a modular power converter provides the capability to manage the thermal stress of the components. This solution, aided by an advanced 


\section{MODULAR CONVERTERS FEATURES}

$\checkmark$ Inherent fault tolerant capability

$\checkmark$ Better quality in the output waveforms

$\checkmark$ Reduced output filters

$\checkmark$ Fast PEBB replacement

$\checkmark$ Standarization in the PEBB design

$\checkmark$ Reduction in the design costs using the same PEBB for different applications

Reduced maintenance costs if a proper RUL management method is implemented!

× Complex hardware/software design × Large number of components and sensors $\boldsymbol{X}$ Large computational burden for the estimation of RUL $\times$ Requirement of fault diagnosis methods

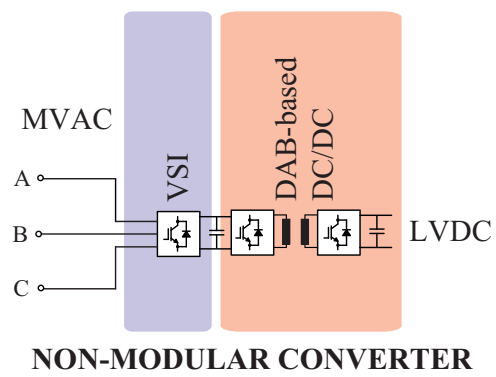

a)

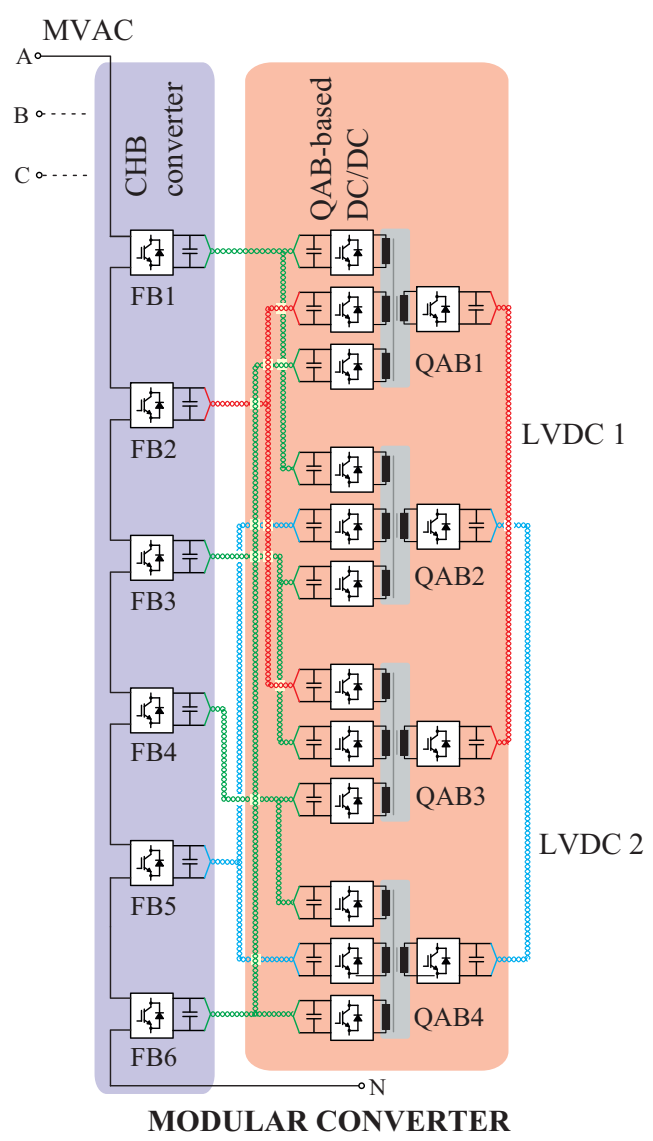

b)

Figure 1: Impact of a failure on a power converter a) considering a non-modular system consisting of a voltage source inverter (VSI) and a dc/dc dual active bridge converter (DAB) b) considering a modular power converter consisting of a six-cell cascaded H-bridge converter (CHB) and four quadruple active bridge converters (QAB)

condition monitoring and carried out by proper control strategies, allows keeping the advantages of the modular converters with the added capability of managing the RUL according to external constraints, i.e., the maintenance schedule. This manuscript has overviewed a series of techniques that make the operation of modular converters smarter, having the reliable operation as a design parameter. These techniques could be the foundation of a new paradigm in the power converters field that has been emerging in the recent years.

\section{Power Converters Reliability Issues}

In the field of power electronics reliability a paradigm-shift has taken place due to the extremely high reliability requirements present in many applications, like automotive or aerospace: use the physic of failure methodologies developed in the last 50 years in the design and control of power electronics [21]. Failure mechanisms need to be understood and need to be taken into account in the design of the system along with the lifetime target. Power components failures knowledge and the expected real operating conditions of the device are being taken into account 
during the design process [22]. These estimated real conditions are included in an expected mission profile that includes relevant stressor like temperature, vibration, humidity and others.

In the power converters, the most sensitive components in the system are reported to be the power semiconductors and the capacitors. These components are usually the source of more than $50 \%$ of unscheduled maintenance cost [23]-[25]. The wide range of nominal power of the converters induces to focus the attention in different power devices to extend the expected lifetime. Power converters for low voltage high current applications may see their lifetime reduced by output capacitors, while for grid interface converters at higher voltages, the power modules are usually regarded as more critical [25]. For power semiconductors, thermal stress by means of thermal cycles (heating-cooling or vice versa) and presence of high temperatures have been identified to be the main cause for device aging and final failure. The most important failure mechanisms of power semiconductors are bond wire lift off, chip solder fatigue and base-plate solder fatigue, all caused by thermal stress [26]. In the case of power capacitors, the capacitor type has impact on the relevant failure mechanisms, but a common stressor is also the high temperature. A reduction of the hotspot temperature of the capacitor increases the lifetime of the device [27].

The detailed knowledge of the failure mechanism in power electronics components such as power modules and capacitors allows to introduce also in the field of power electronics the predictive maintenance [47] already adopted for several other power system equipment. On the basis of aging indicators and estimating the remaining useful lifetime (RUL) maintenance can be accurately schedule minimizing the already highlighted costs of unscheduled maintenance.

Condition monitoring of power semiconductors focuses on aging indicators such as the collector-emitter saturation voltage, gate-emitter threshold voltage, short circuit current, gate current, turn-on and off times, power device thermal resistance and current-change rate [28]. Various online and offline condition monitoring techniques have been established to monitor these parameters to define the health-status of the power device [29]. Apart from these aging indicators, lifetime models are also employed to predict the failures when the converter is working under different operating conditions. However, parameter sensitivity to degradation of the devices, measurement complexity and degree of uncertainty in the state of health estimation remain as a challenge.

For the capacitors, the main RUL indicators are the equivalent series resistance (ESR), capacitance, ripple voltage, volume and temperature [30]. One common methodology is to sense the capacitor ripple current to obtain the ESR or capacitance either with or without signal injection. In order to avoid additional current sensors, model-based methods estimate the capacitance from the converter model. More advanced data driven methods are also proposed to estimate the capacitance using available signals such as input/output voltages and current, dc-link voltage, etcetera [31]. As a summary, it can be affirmed that the capacitor hotspot temperature and semiconductor junction temperature are critical parameters to achieve an accurate estimation of their RUL. These parameters can be obtained by:

1) Model based, from the converter operating parameters.

2) Combination of model and additional sensors (for example to estimate the capacitors equivalent series resistance ESR).

3) Embedded sensors in the capacitors and extra voltage sensors to measure the thermo-sensitive electrical parameters (TSEP) of the power switches, like the on-state collector-emitter $V_{c e}$ voltage. 


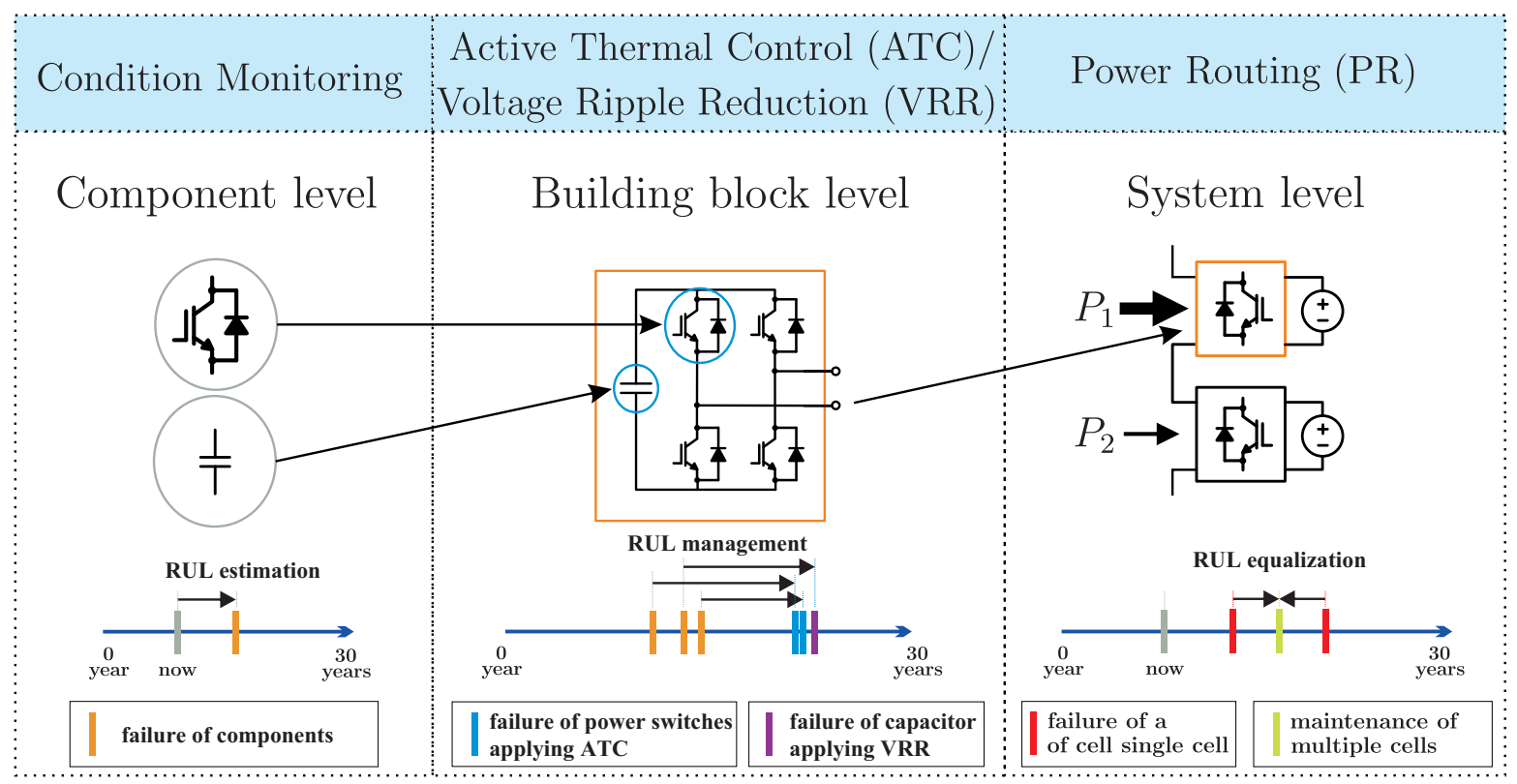

Figure 2: Application of ATC and VRR methods to achieve the RUL management and power routing for system level to facilitate the scheduled maintenance

\section{Maintenance Scheduling Management in Modular Converters}

Maintenance tasks related to power systems usually require trained personnel with expertise, and according to the level of failure, the system downtime may vary. Unscheduled maintenance can result in revenue loss and unavailability of the systems and these facts become crucial with increasing operational demands and lack of financial resources. Therefore, prognostic maintenance has become an essential requirement for systems requiring high reliability and availability.

A definition of prognostics is the ability to provide early detection of the incipient faults of a component and to predict the progression of such a fault resulting to the component failure [32]. With prognostics, the system RUL can be predicted with an acceptable degree of confidence and when such an information is used to schedule the maintenance, it is termed as prognostic maintenance [33].

As challenges for modular power conversion systems, replacements of building blocks, device-to device parameter mismatching and different impact of the cooling system result in a significant difference in the PEBBs RUL in the system. The first step to determine the RUL of all sensitive components in a power conversion system is to use a condition monitoring system that is applied to determine the average time where each component will fail, represented with orange bars in Fig. 2.

The estimation of the components RUL in each PEBB can be taken into account to apply a RUL management method for the PEBB. For power semiconductors, the active thermal control (ATC) method has been proposed, which targets to regulate the thermal-mechanical stress. ATC methods are able to manage the rate of change of the power semiconductors degradation by preventing over-temperatures and managing the power cycling obtaining a 
reduction in the thermal stress [34], [35]. For the capacitors, the voltage ripple reduction (VRR) method has been proposed to reduce their hotspot temperature. VRR can be achieved in several ways: a) At the design phase by adding more capacitors in parallel, with the disadvantage of increasing the cost b) Reducing the current of that capacitor, for example by affecting the power transfer of the cells connected to that capacitor. c) Employing active methods implemented by advanced control and modulation methods [36]-[38].

The combination of ATC and VRR takes into account the most relevant failure mechanisms of the most sensitive components. The application of these methods makes possible the smart management the RUL of the PEBBs. This fact is shown in Fig. 2 with blue and purple bars, where the ATC and VRR methods, at PEBB level, use the information provided by the condition monitoring to extend as much as possible each PEBB RUL. It is expected that with the improvement of the RUL estimation method, the efficacy of these techniques will improve. However, even with an estimation that is affected by inaccuracies, putting the reliability at the center of the power electronics design and control, still allows for a reduction in the spread of the failures, with benefits in terms of maintenance scheduling.

Several researchers have focused their efforts on optimizing the maintenance schedule based on the monitored data considering operational constraints using the previously introduced power devices condition monitoring [39], [40]. However, literature discussing about actively influencing the RUL and thereby optimizing the maintenance scheduling is scarce. In fact, the maintenance optimization can have multiple conflicting objectives such as reducing the number of maintenance, maximum utilization of RUL of the components, scheduling the maintenance according to the availability of personnel, material, etcetera. In the conventional method, prognostic maintenance with condition monitoring schedules the maintenance operations when the probability of failure reaches a threshold value, and the PEBBs that are close to fail before the next possible maintenance are replaced. This results in a loss of RUL of the PEBBs which could potentially operate for longer time.

ATC methods through power routing and VRR methods have the capability to manipulate the RUL of the system to achieve these conflicting goals more efficiently [41], [42]. The main novel concept of the power routing is that the equal power sharing across the cells in a modular converter is not always the optimal solution. In case of having power cells with unequal aging, the application of an unbalanced power sharing between the cells by applying a power routing method achieves important maintenance goals. The application of power routing control allows optimizing at system level the maintenance schedule minimizing the cost leading to the maximum operation profit.

As commented previously, the power routing method is based on a flexible power sharing between the PEBBs of the power converter taking into account the actual estimated values of the RUL of each PEBB. In this way, power routing not only have to take into account instantaneous electric measurements but also the knowledge of the previous operational conditions of the power system. For instance, in data centers the power routing implementation is done based on energy consumption (kWh and instantaneous current) [43].

Remarkably, power routing can be implemented in parallel building blocks as well as in series connected building blocks [44], [45]. This concept is shown in Fig. 2 where the power routing is applied in order to achieve a RUL equalization between the PEBBs. This method considers that, looking for the optimization of the maintenance schedule, it is interesting to equalize all the PEBB RUL at the exact maintenance time [36]. With an active RUL 


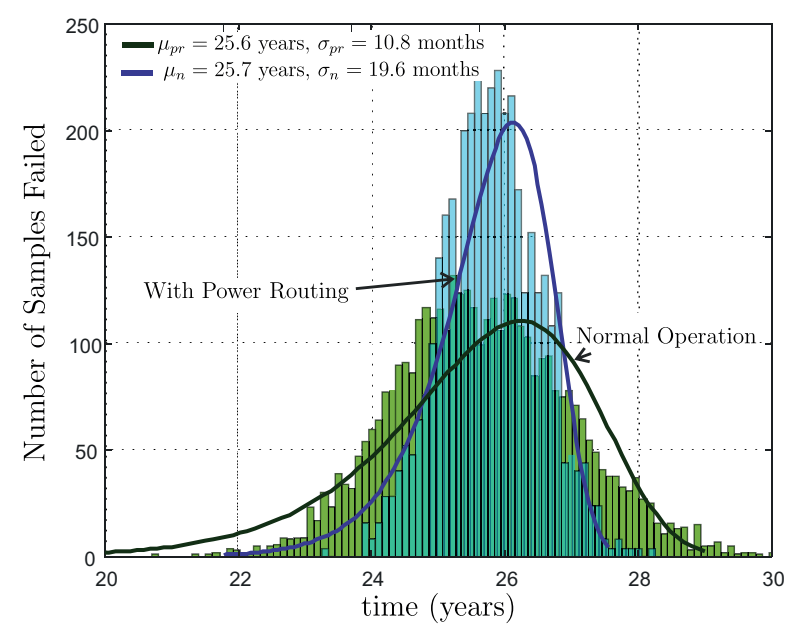

(a)

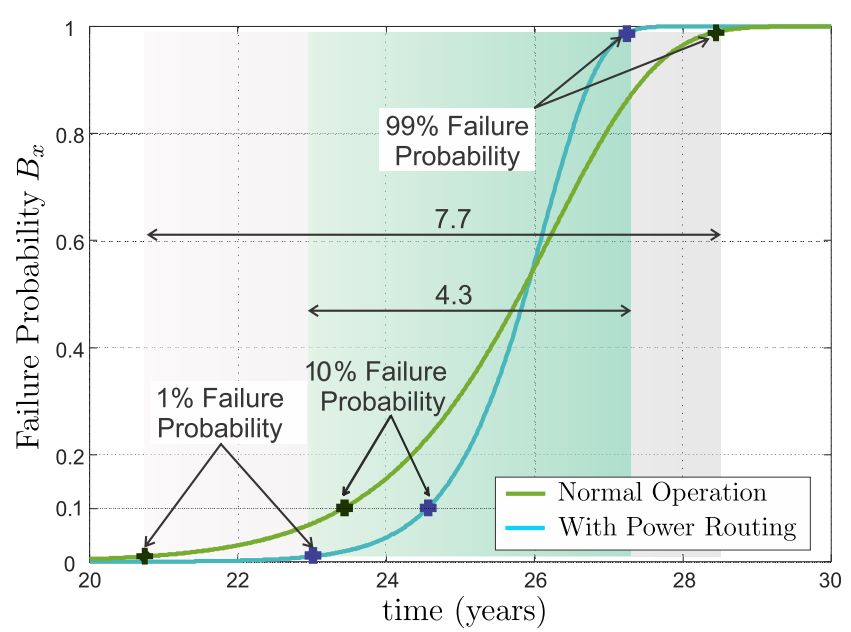

(b)

Figure 3: Monte Carlo analysis without and with lifetime management applying power routing (a) Distribution of failures over time (b) Unreliability or Cumulative probability distribution over time [42].

control via power routing that considers the maintenance schedule, the maintenance can be planned without wasting significant RUL of certain components. This idea is represented in Fig. 2, where the expected time of failure of each PEBB, represented with red bars are modified by the power routing method in order to merge them in only one instant represented with a green bar.

It is important to notice that, system parameters such as the collector-emitter voltage $\left(V_{c e}\right)$ and turn-on/turn-off energies of the devices are subjected to device-to-device parameter mismatch. In addition, the thermal coupling creates temperature differences in the components and also non-uniform cooling systems provoke temperature differences among the components. As an example, the datasheet of power devices indicate a variation up to $20 \%$ from the mean value for $V_{c e}$ [46].

In order to evaluate the performance of the power routing method incorporating the parameter mismatch, a Monte-Carlo analysis [42] can be done with and without the power routing control. This analysis considers the unavoidable parameter mismatch with suitable probabilistic distributions, such as the Gaussian distribution. The impact on a modular converter RUL is studied with electro-thermal models generating the estimated power devices junction temperatures for the selected long-term mission profile. Finally, the RUL estimations are based on the state-of-the-art lifetime models using rainflow counting [47], [48].

As an example, a modular DAB system with four input-parallel output-parallel converters for a more electric aircraft application is considered in the Monte-Carlo analysis. In Fig. 3a), the results obtained from this study are summarized representing a histogram that represents the number of times that the converter failed at some specific instant.

The analysis of the obtained results derives on the determination of the factor $B_{x}$ that represents the time where the system has a probability of $x \%$ of failure. Remarkably, this methodology assigns a probability of failure, which 


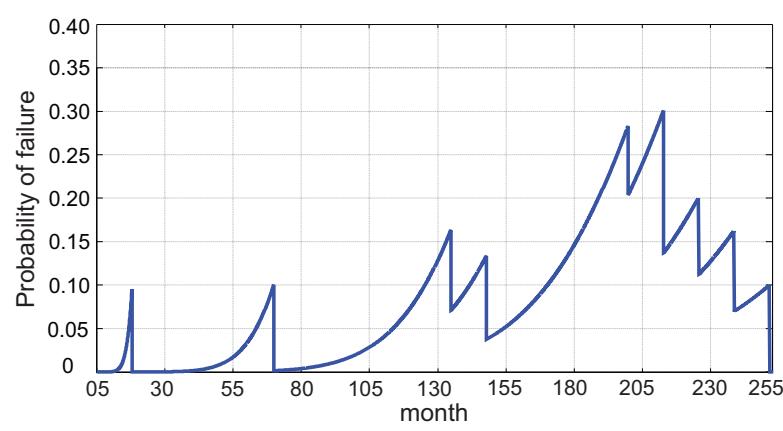

a)

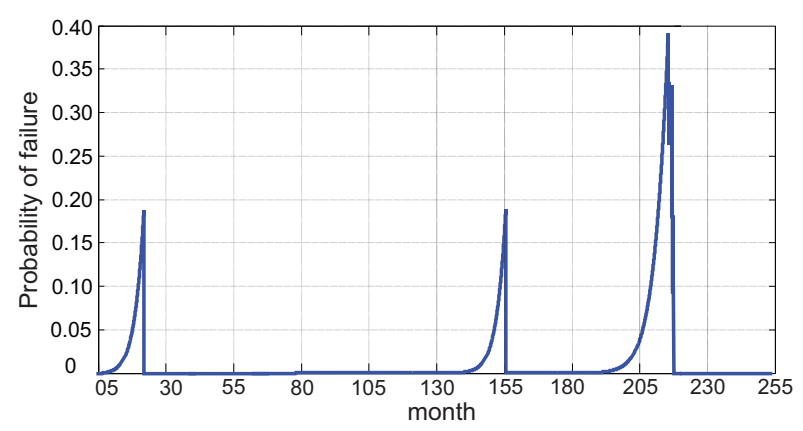

b)

Figure 4: Probability of failure in a PEBB-based modular converter operating as Smart Transformer (a) normal operation (b) with power routing for maintenance optimization [49]

is dependent on the previous operation and thereby differs from the constant failure rate assumption. In Fig. 3b), the representation of the failure probability $B_{x}$ taken from the Monte-Carlo analysis is shown. Without any active RUL control, the system reaches the values $B_{0.1}, B_{1}$ and $B_{10}$ around 18.43, 20.75 and 23.45 years respectively [42]. On the other hand, when the power routing control is employed, the system attains the factors $B_{0.1}, B_{1}$ and $B_{10}$ around 20.75, 22.95 and 24.65 and years, respectively, resulting in a meaningful prolongation of the RUL, as shown in Fig. 3b). Depending on the application and the corresponding availability requirement, the probability of failure to consider the replacement of PEBBs are defined. For instance, in aerospace applications where the safety is critical issue, factors as $B_{0.1}, B_{1}$ can be considered eligible to be used to warn the PEBB replacement.

In addition to the lifetime extension achieved by the power routing method, another important feature can be also highlighted. In case of modular systems with many converter PEBBs, a single maintenance to replace all the cells appears to be impractical, especially if the components RUL is spread. From the results in Fig. 3a), approximating the histogram envelope with a Gaussian waveform, it can be observed that the average time to failure $(\mu)$ is very similar applying and not applying the power routing method (25.65 years approximately). However, it can be observed that the power routing control is able to achieve a $44 \%$ reduction in the standard deviation of failures $(\sigma)$ compared to that of normal operation. This feature can be also clearly observed in Fig. 3b) where it is can be seen that, applying the power routing control, $98 \%$ of failures are reduced from 7.7 up to 4.3 years. This represents an advantage from the maintenance scheduling point of view reducing the probability of spread PEBB failures. This fact increases the possibility to minimize the maintenance tasks by replacing all the PEBBs at the same time.

As another example to show this advantage, the control strategy focused on scheduling the maintenance while maximizing the utilization of the RUL of individual converter PEBBs for a smart transformer (ST) application has been analyzed [49]. Considering the fact that even the lifetime models are developed by curve fitting of the power cycling test results, the variability in the lifetime model motivates to represent the RUL or time to failure as a probability density function. The probability of failure incorporating the variability in the lifetime model is obtained through Monte-Carlo simulations considering the curve-fitting parameters in lifetime model as Gaussian distributions. The probability of failure of a modular converter with ten unequally aged PEBBs working as a ST 
without applying power routing is illustrated in Fig. 4a). In this experiment, it is assumed that the individual PEBBs are replaced when they reach a probability of failure equal to $10 \%$. The probability of failure of the overall modular converter shows that there is a high chance of failure in the system spread over years, which can significantly affect the availability of the ST.

On the other hand, Fig. 4b) shows the probability of failure of the modular converter with power routing control by setting the same individual PEBB replacement criterion. In order to minimize the maintenance cost, the objective of the power routing control method is to force the substitution of multiple PEBBs at the same time. This can be done by managing the RUL of the PEBBs that are close to reach the probability of failure threshold within the next maintenance interval. Compared to the failure probability with normal operation, applying the power routing method with management of maintenance scheduling causes a concentrated probability of failure around only three maintenance instants obtaining a reduction in the maintenance cost.

It is true that the maintenance schedule depends on many issues and usually it is periodically scheduled since it covers not only electrical issues but also ambient and mechanical conditions. Anyway, the power routing method is also useful when a programmed maintenance is going to be carried out. In this case, the power routing technique can take this information into account managing the PEBBs RUL in order to squeeze the remaining lifetime of those PEBBs that are close to achieve the replacement threshold. This strategy actually saves some lifetime of the remaining PEBBs improving the overall lifetime of the power system.

\section{Implementation of POWER Routing in MOdular Converters}

As described previously, the power routing technique can be applied to modular dc/dc or dc/ac converters in order to manage the power devices temperatures and consequently their RUL. In dc/dc converters working with output parallel connection, when the power routing concept is applied the power managed by each PEBB is different, generating different output currents in the PEBBs. Since the power devices losses are mainly dependent on the current, the power routing in the paralleled dc/dc converters facilitates the management of the power devices RUL. On the other hand, in modular dc/ac converters such as the CHB, each phase voltages is composed by the addition of the PEBBs voltages because they are connected in series. When the power routing technique is applied to the $\mathrm{CHB}$, the voltage generated by each PEBB is different, while the same phase current is flowing through all PEBBs. Therefore, both modular series and parallel connected PEBB-based converters are dual. When the power routing technique is applied, the voltage or the current are determined by the sum of unbalanced voltages and currents.

The power routing forces a non-balanced power among the PEBBs, while the total power of the system remains usually constant. In the case of modular dc/dc converters, a possibility to apply the power routing method is to implement a closed-loop controller that takes into account the state of health $(\mathrm{SoH})$ of the power cells to optimize the maintenance cost. An example of the type of strategy is shown in Fig. 5 [50]. The SoH uses the knowledge about the previous operation of the power system and the instantaneous electrical measurements to determine the aging of each cell. This information is taken into account by the maintenance cost optimizer that determines the most proper power sharing between the modules $\left(k_{n}\right)$. The total power of the overall converter $P$ is shared among the power cells (with input and output de voltages denoted by $V_{d}$ and $V_{o}$ respectively, and $I_{n}$ is the inductor current) 
considering the information provided by the maintenance optimizer and the power to be managed by each cell $P_{n}$ is finally obtained by a conventional dedicated controller in the cell. The application of this method in a modular $\mathrm{dc} / \mathrm{dc}$ converter permits to manage the temperature of the cells extending the overall converter lifetime [51].

On the other hand, in order to implement the power routing in the modular series converters, the approach based on applying modified modulation strategies is the most promising way, since they enable to individually manipulate the power losses, and consequently the thermal stress of each PEBB. The usual way to operate modular series or parallel converters is the phase-shifted PWM (PS-PWM) technique [52], [53]. In fact the well-known interleaved operation of parallel dc/dc converters is a particular case of the PS-PWM method. As an example to include the power routing techniques in the modulation strategy based on the PS-PWM technique, several modulation methods applied to the CHB can be addressed (see Fig. 6): a) the modulation technique based on pure sinusoidal references, b) the multi-frequency modulation method, and c) the discontinuous modulation technique.

The modulation method based on sinusoidal voltage reference waveforms is very simple. This modulation method modifies the modulation index of each PEBB as shown in Fig. 6a. However, the power routing capability, that is the possibility to force a power imbalance between the PEBBs, is highly limited by the over-modulation in the PEBBs. In order to tackle this problem, other strategies such as the multi-frequency modulation technique (Fig. 6b) [54] or the discontinuous modulation method (Fig. 6c) have been proposed [45], [55].

In the multi-frequency modulation strategy, the third-harmonic injection concept is applied to the PEBBs duty cycles, allowing to extend the power routing capability of the system by means of the extended linear modulation region. Remarkably, two variants of the modulation signal are utilized in order to make this technique feasible to be applied to single-phase inverters. However, this method offers a limited capability to handle the thermal stress of PEBBs connected in series. This is because the phase current flowing through them is not affected (only the current of parallel PEBBs is changed) by the power routing, causing the limited variation of thermal stress of series PEBBs.

Discontinuous modulation uses two variants of modulation signal: clamped signal to force an additional loading power in a PEBB and non-clamped signal to relax the PEBB loading power. The basic principle to perform the power routing with this modulation strategy is based on determining the clamping angle $\phi$. The most interesting feature of this method is the ability to efficiently manage the thermal stress of series PEBBs, since their losses are varied by the clamped signal, achieving the power routing as well.

The major characteristics of three modulation techniques are summarized in Table I. The multi-frequency and discontinuous methods present improved power routing capability compared to the pure sinusoidal method. On the other hand, only the discontinuous method is able to manage the thermal stress of both parallel and series PEBBs, whereas others can handle the thermal stress of only the parallel PEBBs.

\section{Method to Mitigate the Collateral negative Effects of the Modulation Approaches to IMPLEMENT POWER ROUTING}

The implementation of the power routing with the modulation methods introduced in the previous section achieves the objectives of the power routing improving the maintenance scheduling. However, the application of these methods 


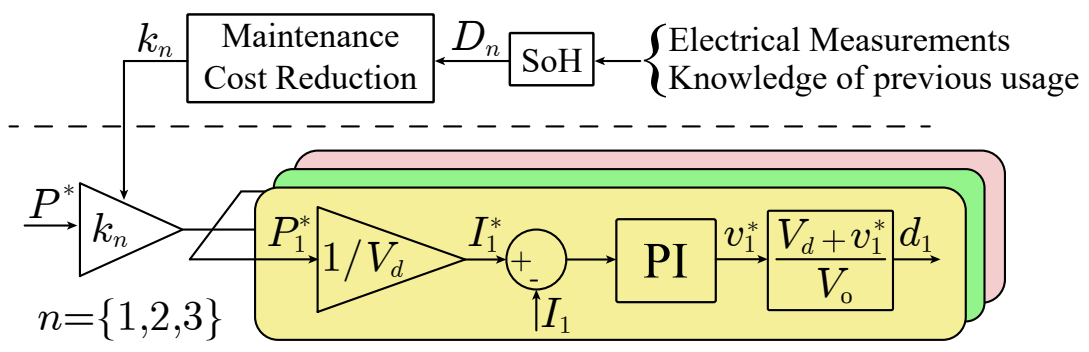

Figure 5: Control strategy to implement the power routing method in a parallel-connected modular dc/dc converter

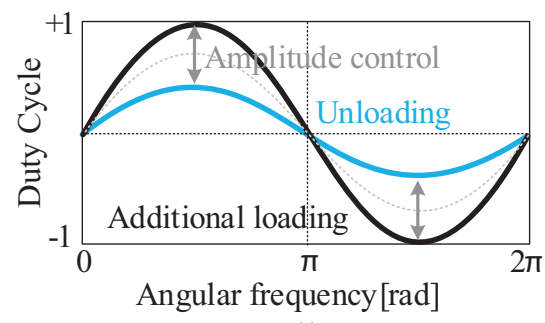

(a)

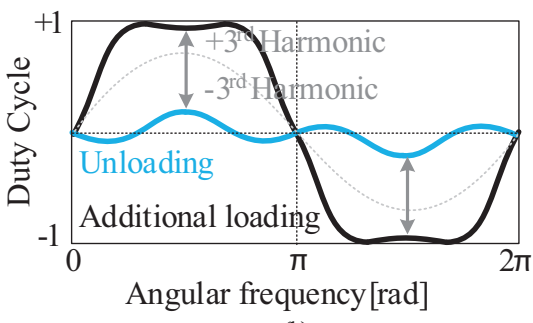

(b)

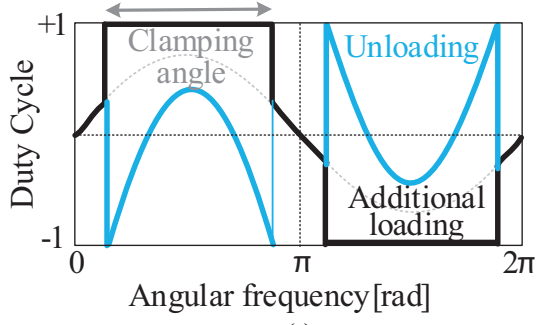

(c)

Figure 6: Strategies to implement ATC via power routing: (a) sinusoidal modulation (b) multi-frequency modulation (c) discontinuous modulation

TABLE I: Comparison of different modulation methods to implement power routing in the modular converter shown in Fig. 1b

\begin{tabular}{|c|c|c|}
\hline Modulation Method & $\begin{array}{c}\text { Power Routing } \\
\text { Management Capability }\end{array}$ & $\begin{array}{c}\text { Thermal stress } \\
\text { Management capability }\end{array}$ \\
\hline Pure sinusoidal & ( Limited by overmodulation & $\begin{array}{l}\Theta \text { Mainly available for parallel PEBBs, } \\
\text { highly limited for series PEBBs }\end{array}$ \\
\hline Multi-frequency & $\begin{array}{l}15.5 \% \text { improvement compared } \\
\text { to the pure sinusoidal method }\end{array}$ & $\begin{array}{l}\Theta \text { Mainly available for parallel PEBBs, } \\
\text { highly limited for series PEBBs }\end{array}$ \\
\hline Discontinuous & $\begin{array}{l}\text { (1) Similar or slightly better } \\
\text { than the multi-frequency method }\end{array}$ & (1) Available for both parallel and series PEBBs \\
\hline
\end{tabular}

presents collateral negative effects in the converter operation.

The PS-PWM method is a high-performance solution to operate modular converters, but only if all PEBBs manage the same power. When the power routing concept is applied, the conventional PS-PWM method loses, partially, its high performance. This phenomenon can be observed in Fig. 7, where a three-cell CHB and a three-module interleaved dc/dc converters are tested.

In the case of the CHB results, the CHB is formed by three power cells with de voltage equal to 150 volts and modulation index equal to 0.8. A balanced operation is forced during the first $40 \mathrm{~ms}$ generating the high performance output voltage shown in Fig. 7a with the superior harmonic performance represented in Fig. 7b. From $t=40 \mathrm{~ms}$, a power routing method by implementing a discontinuous modulation method is applied as shown 

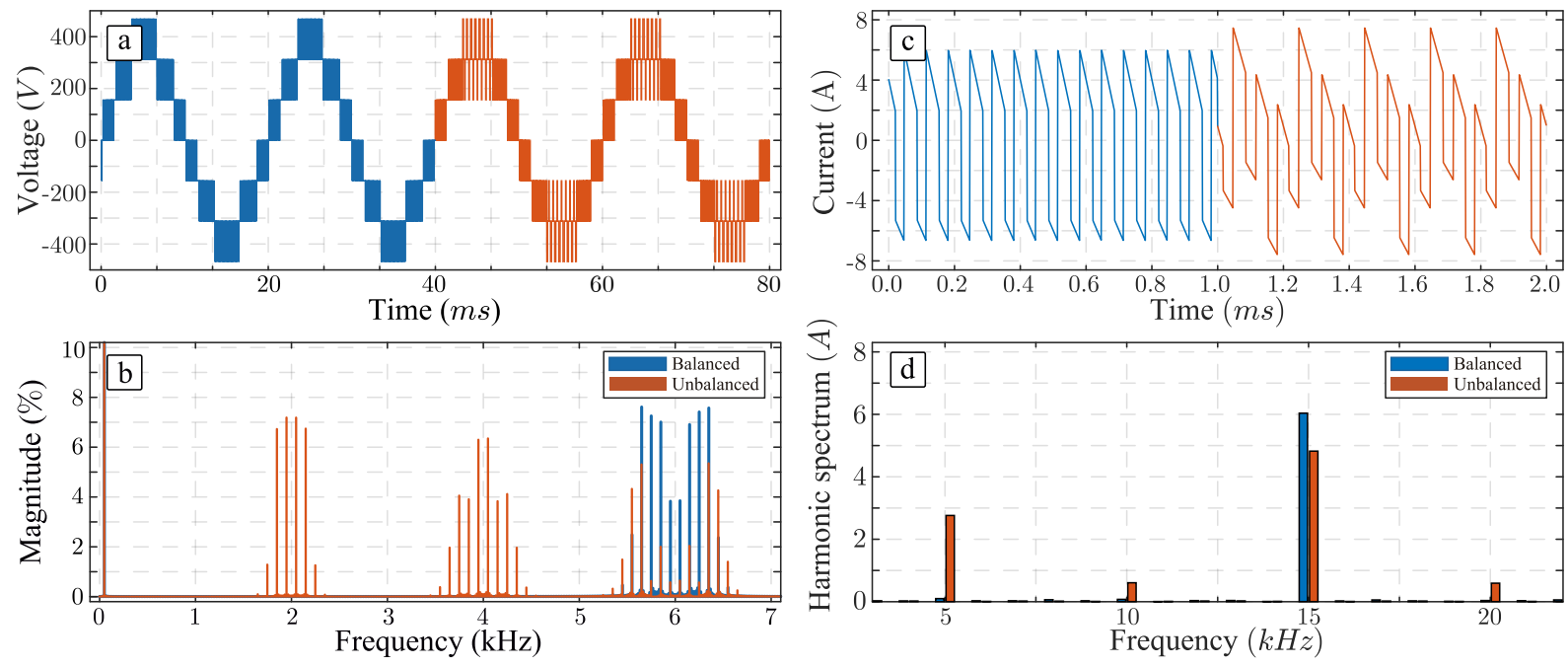

Figure 7: Example of unbalanced operation in modular converters and its consequences a) CHB output voltage b) CHB output voltage harmonic spectrum c) Interleaved $\mathrm{dc} / \mathrm{dc}$ boost converter output capacitor current d) Interleaved dc/dc boost converter output capacitor current harmonic spectrum

in Fig. 6c. The application of this power routing method permits to modify the average temperature of the cells permitting to save lifetime of the clamped cell extending the lifetime of the overall converter [56]. However, as it can be clearly observed in Fig. 7b, the harmonic performance is degraded by the appearance of carrier-frequency order harmonic distortion.

A similar conclusion can be obtained from the results considering the modular $\mathrm{dc} / \mathrm{dc}$ boost converter. In this case, initially the power managed by each boost converter is $1.6 \mathrm{~kW}$ and the interleaved operation presents a superior harmonic performance of the current through the output capacitor as shown in Fig. 7d. However, from $t=1 \mathrm{~ms}$, the power is not equally shared imposing a power sharing equal to $1.6,0.75$ and $1.24 \mathrm{~kW}$. The cells managing lower power decrease their average temperatures inducing a lower aging [51]. However, the power imbalance forced by the power routing method provokes the degradation of the harmonic performance of the interleaved converter as can be also observed in Fig. 7d.

As a summary, it can be observed that, due to the unbalanced operation of the modular converters, a harmonic distortion appears at the switching frequency $f_{c}$ (for the $\mathrm{dc} / \mathrm{dc}$ converters operated with bipolar PWM in each PEBB) or at $2 f_{c}$ (for the CHB operated with unipolar PWM in each PEBB). On one hand, this phenomenon in serial-connected modular converters such as the CHB generates distorted output voltages and consequently the output filter has to be redesigned in order to keep the minimum output waveforms quality. On the other hand, in parallel-connected modular converters such as the interleaved dc/dc boost topology, the low frequency harmonic distortion in the output current created by the unbalanced operation creates an increase in the output capacitor temperature provoking a reduction in its expected RUL.

The effects provoked by the unbalanced operation forced by the power routing in modular converters (series or parallel connected) can be partially mitigated by applying advance modulation methods which apply the PS- 


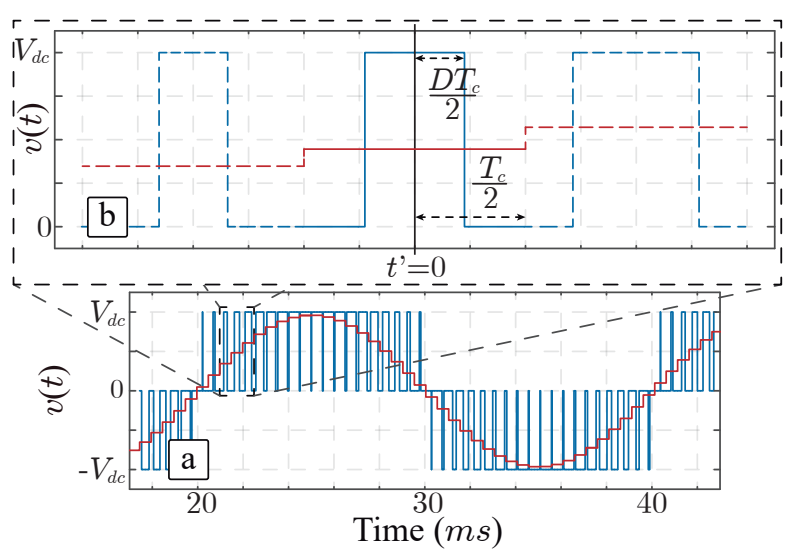

Figure 8: H-bridge output voltage. a) A complete $50 \mathrm{~Hz}$ period b) Single sampling time period

PWM method but with using non-fixed phase displacement angles. These methods, called variable-angle PS-PWM techniques, consider that the converter output waveforms (voltages and currents) are periodic signals and therefore can be described by a Fourier Series determining its Fourier coefficients. This harmonic description can be based on fundamental frequency or switching frequency as shown in Fig. 8 where the output voltage waveform of a full bridge PEBB belonging to a CHB is represented.

Variable-angle PS-PWM methods are focused on eliminating or reducing the harmonic distortion below $N$ times $f_{c}$, mitigating the negative effects of the unbalanced operation of the converter forced by the power routing technique in order to manage the power devices RUL. The mathematical description of the output waveforms is the base to achieve this goal helping to determine the phase displacement angles $\phi_{i}$ that minimize the low frequency distortion.

The angle solution set to eliminate or mitigate some particular harmonic content can be easily obtained through an analytic approach for modular converters composed by three PEBBs [56]-[58]. However, as the number of PEBBs grows, the analytical solution to mitigate the negative effects of power routing can be hard to obtain or even does not exist because these solutions are highly depending on the number of PEBBs [59].

In this sense, it is necessary to find and define new techniques to obtain a particular angle solution set that fulfills the requirements. Considering the mathematical harmonic description for these systems, the distortion provoked by power routing can be eliminated, minimized or even re-shaped formulating the mathematical problem as a flexible multi-objective multi-variable cost function. The computing capability of the controller systems has experienced a huge growth in the last decade. In this way, personal computers, server-based solutions, multi-core low-cost microcontrollers, FPGA, System of Chip (SoC) and cloud-based solutions currently overcome the computational requirements and they are available in the manufactures portfolio with a very competitive price. This fact makes that numerical computation approaches, advanced metaheuristic searching algorithms or artificial intelligent can be used to face out this kind of multi-objective and multi-variable mathematical problems in real-time [60], [61].

The application of the variable-angle PS-PWM has been tested in both modular converters, series connected such as the CHB or parallel connected such as the interleaved dc/dc converters. In Fig. 9, the obtained results for both series and parallel modular converters are shown. In the figure, the power converters output waveforms and the 

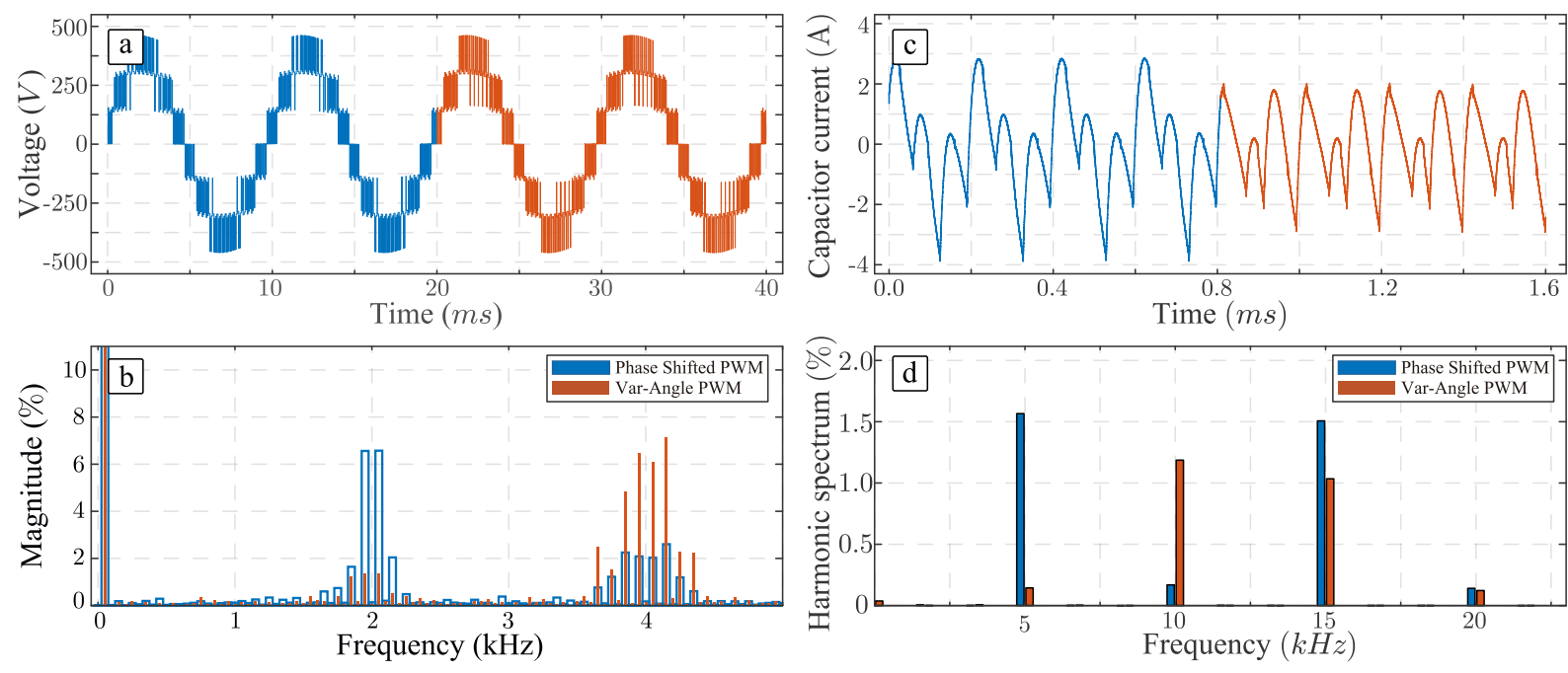

Figure 9: Comparison between traditional PS-PWM and variable-angle PS-PWM operation for modular converters.

a) $\mathrm{CHB}$ output voltage. b) $\mathrm{CHB}$ output voltage spectrum. c) Interleaved dc/dc boost converter output current through capacitor. d) Interleaved dc/dc boost converter capacitor current harmonic spectrum

corresponding harmonic spectra are represented. It can be seen that the application of the variable-angle PS-PWM methods improves the output waveform quality reducing the output filter stage in the CHB [56], [57] and decreasing the output capacitor average hotspot temperature in the interleaved dc/dc boost converter giving rise to achieve an extension of its lifetime [62].

\section{CONCLUSION}

This paper advocates for an increased intelligence of the power electronics of modular systems. The goal is to include the end-of-life or the maintenance schedule in the actual operations of the converter. To accomplish this goal, there are several conditions to fulfill:

- Condition monitoring for the components is implemented in all PEBBs, to determine the remaining useful lifetime.

- It is possible to control the power processed by the PEBBs in the modular structure (power routing).

- The performance of the system shall not be degraded because of this modified operation.

- The overall cost of the system should not be impacted by the additional functionalities

The two main methods that make possible this intelligent operation mode are the active thermal control and the thermally-compensated modulation techniques. Active thermal control allows changing the electrical parameters of the power converter to modify the component stress. In particular, the power routing technique shifts the power among the PEBBs composing the modular converter in order to affect their individual lifetime. In this way, the probability of failure can be modified to locate the failure at a specific instant in time allowing to simplify the maintenance scheduling and reducing the corresponding cost. 
In order to avoid the performance degradation in terms of power quality when the power routing is applied, advanced modulation techniques based on the adaptive phase shifting of the PWM carriers of the PEBBs can be implemented. Despite asymmetric operations, the harmonic spectrum still retains very low total harmonic distortion.

To conclude, the power routing in conjunction with adaptive carrier phase shifting PWM constitute a technology combination, which has the impact of improving the reliability of power electronics systems without performance deterioration with a minimum additional initial cost.

Future research on the topic at all levels is envisaged to further enhance the scope of the proposal, i.e.:

- Component level: including more components, i.e., gate drivers and pcb, in the condition monitoring.

- Topology level: evaluating the best topologies to take fully advantage of the power routing concept.

- System level: optimizing the maintenance scheduling taking into consideration actual variabilities in real word applying new approaches such as artificial intelligence, internet of things technology, big data, neural networks, etcetera.

\section{REFERENCES}

[1] M. Liserre, T. Sauter, and J. Y. Hung, "Future energy systems: Integrating renewable energy sources into the smart power grid through industrial electronics," IEEE industrial electronics magazine, vol. 4, no. 1, pp. 18-37, 2010.

[2] Z. Cheng, J. Duan, and M. Chow, "To centralize or to distribute: That is the question: A comparison of advanced microgrid management systems," IEEE Industrial Electronics Magazine, vol. 12, no. 1, pp. 6-24, March 2018.

[3] W. Luo, L. G. Franquelo, M. Malinowski, S. Stynski, D. Vinnikov, and A. Chub, "Energy storage systems for power grid utilities - part i," IEEE Industrial Electronics Magazine, vol. 14, no. 1, March 2020.

[4] _ _ "Energy storage systems for power grid utilities - part ii," IEEE Industrial Electronics Magazine, vol. 14, no. 1, March 2020.

[5] J. I. Leon, E. Dominguez, L. Wu, A. Marquez, M. Reyes, and J. Liu, "Hybrid energy storage systems: Concept, advantages and applications," IEEE Industrial Electronics Magazine, vol. 14, no. 1, March 2020.

[6] G. Buticchi, S. Bozhko, M. Liserre, P. Wheeler, and K. Al-Haddad, "On-board microgrids for the more electric aircraft—-technology review," IEEE Transactions on Industrial Electronics, vol. 66, no. 7, pp. 5588-5599, July 2019.

[7] J. A. P. Lopes, F. J. Soares, and P. M. R. Almeida, "Integration of electric vehicles in the electric power system," Proceedings of the IEEE, vol. 99, no. 1, pp. 168-183, 2010.

[8] B. K. Bose, "Power electronics, smart grid, and renewable energy systems," Proceedings of the IEEE, vol. 105, no. 11, pp. 2011-2018, Nov 2017.

[9] J. Endrenyi and G. J. Anders, "Aging, maintenance, and reliability - approaches to preserving equipment health and extending equipment life," IEEE Power and Energy Magazine, vol. 4, no. 3, pp. 59-67, May 2006.

[10] Y. Yang, H. Wang, A. Sangwongwanich, and F. Blaabjerg, "Design for reliability of power electronic systems," in Power Electronics Handbook. Elsevier, 2018, pp. 1423-1440.

[11] J. Endrenyi, S. Aboresheid, R. N. Allan, G. J. Anders, S. Asgarpoor, R. Billinton, N. Chowdhury, E. N. Dialynas, M. Fipper, R. H. Fletcher, C. Grigg, J. McCalley, S. Meliopoulos, T. C. Mielnik, P. Nitu, N. Rau, N. D. Reppen, L. Salvaderi, A. Schneider, and C. Singh, "The present status of maintenance strategies and the impact of maintenance on reliability," IEEE Transactions on Power Systems, vol. 16, no. 4, pp. 638-646, Nov 2001.

[12] C. V. Pop, A. Buzo, G. Pelz, H. Cucu, and C. Burileanu, "The estimation of the lifetime variation for power devices," IEEE Transactions on Device and Materials Reliability, pp. 1-1, 2019.

[13] W. Zhang, D. Xu, P. N. Enjeti, H. Li, J. T. Hawke, and H. S. Krishnamoorthy, "Survey on fault-tolerant techniques for power electronic converters," IEEE Transactions on Power Electronics, vol. 29, no. 12, pp. 6319-6331, Dec 2014.

[14] K. Shenai, "Power electronic module: Enabling the 21st-century energy economy," IEEE Power Electronics Magazine, vol. 1, no. 3, pp. 27-32, Sep. 2014. 
[15] T. Ericsen, "The second electronic revolution (it's all about control)," IEEE Transactions on Industry Applications, vol. 46, no. 5, pp. 1778-1786, Sep. 2010.

[16] T. Ericsen, N. Hingorani, and Y. Khersonsky, "Pebb - power electronics building blocks from concept to reality," in 2006 Record of Conference Papers - IEEE Industry Applications Society 53rd Annual Petroleum and Chemical Industry Conference, Sep. 2006, pp. 1-7.

[17] E. Topuz, "Reliability and availability basics," IEEE Antennas and Propagation Magazine, vol. 51, no. 5, pp. 231-236, Oct 2009.

[18] Wenyuan Li, E. Vaahedi, and P. Choudhury, "Power system equipment aging," IEEE Power and Energy Magazine, vol. 4, no. 3, pp. 52-58, May 2006.

[19] M. Malinowski, K. Gopakumar, J. Rodriguez, and M. A. Perez, "A survey on cascaded multilevel inverters," IEEE Transactions on Industrial Electronics, vol. 57, no. 7, pp. 2197-2206, July 2010.

[20] P. Lezana, J. Pou, T. A. Meynard, J. Rodriguez, S. Ceballos, and F. Richardeau, "Survey on fault operation on multilevel inverters," IEEE Transactions on Industrial Electronics, vol. 57, no. 7, pp. 2207-2218, July 2010.

[21] H. Wang, M. Liserre, and F. Blaabjerg, "Toward reliable power electronics: Challenges, design tools, and opportunities," IEEE Industrial Electronics Magazine, vol. 7, no. 2, pp. 17-26, June 2013.

[22] M. Pecht and A. Dasgupta, "Physics-of-failure: an approach to reliable product development," in IEEE 1995 International Integrated Reliability Workshop. Final Report, Oct 1995, pp. 1-4.

[23] S. Yang, A. Bryant, P. Mawby, D. Xiang, L. Ran, and P. Tavner, "An industry-based survey of reliability in power electronic converters," IEEE Transactions on Industry Applications, vol. 47, no. 3, pp. 1441-1451, May 2011.

[24] H. Wang, F. Blaabjerg, K. Ma, and R. Wu, "Design for reliability in power electronics in renewable energy systems - status and future," in 4th International Conference on Power Engineering, Energy and Electrical Drives, May 2013, pp. 1846-1851.

[25] J. Falck, C. Felgemacher, A. Rojko, M. Liserre, and P. Zacharias, "Reliability of power electronic systems: An industry perspective," IEEE Industrial Electronics Magazine, vol. 12, no. 2, pp. 24-35, June 2018.

[26] V. A. Sankaran, C. Chen, C. S. Avant, and X. Xu, "Power cycling reliability of igbt power modules," in IAS '97. Conference Record of the 1997 IEEE Industry Applications Conference Thirty-Second IAS Annual Meeting, vol. 2, Oct 1997, pp. 1222-1227 vol.2.

[27] W. J. Sarjeant, J. Zirnheld, and F. W. MacDougall, "Capacitors," IEEE Transactions on Plasma Science, vol. 26, no. 5, pp. 1368-1392, Oct 1998.

[28] Y. Avenas, L. Dupont, N. Baker, H. Zara, and F. Barruel, "Condition monitoring: A decade of proposed techniques," IEEE Industrial Electronics Magazine, vol. 9, no. 4, pp. 22-36, Dec 2015.

[29] R. Schmidt and U. Scheuermann, "Using the chip as a temperature sensor - the influence of steep lateral temperature gradients on the vce(t)-measurement," EPE Journal, vol. 21, no. 2, pp. 5-11, 2011. [Online]. Available: https://doi.org/10.1080/09398368.2011.11463790

[30] H. Soliman, H. Wang, and F. Blaabjerg, "A review of the condition monitoring of capacitors in power electronic converters," IEEE Transactions on Industry Applications, vol. 52, no. 6, pp. 4976-4989, Nov 2016.

[31] H. Soliman, H. Wang, B. Gadalla, and F. Blaabjerg, "Condition monitoring for dc-link capacitors based on artificial neural network algorithm," in 2015 IEEE 5th International Conference on Power Engineering, Energy and Electrical Drives (POWERENG), May 2015, pp. 587-591.

[32] F. Camci, "System maintenance scheduling with prognostics information using genetic algorithm," IEEE Transactions on Reliability, vol. 58, no. 3, pp. 539-552, Sept 2009.

[33] Z. Lv, J. Wang, G. Zhang, and H. Jiayang, "Prognostics health management of condition-based maintenance for aircraft engine systems," in 2015 IEEE Conference on Prognostics and Health Management (PHM), June 2015, pp. 1-6.

[34] D. A. Murdock, J. E. R. Torres, J. J. Connors, and R. D. Lorenz, "Active thermal control of power electronic modules," IEEE Transactions on Industry Applications, vol. 42, no. 2, pp. 552-558, March 2006.

[35] M. Andresen, M. Liserre, and G. Buticchi, "Review of active thermal and lifetime control techniques for power electronic modules," in 2014 16th European Conference on Power Electronics and Applications, Aug 2014, pp. 1-10.

[36] M. Andresen, J. Kuprat, V. Raveendran, J. Falck, and M. Liserre, "Active thermal control for delaying maintenance of power electronics converters," Chinese Journal of Electrical Engineering, vol. 4, no. 3, pp. 13-20, Sep. 2018.

[37] S. Wang, R. Teodorescu, and S. K. Chaudhary, "Capacitor voltage ripple reduction methods of modular multilevel converter under unbalanced fault conditions: A comparison," in 2018 IEEE International Power Electronics and Application Conference and Exposition (PEAC), Nov 2018, pp. 1-6. 
[38] R. Picas, S. Ceballos, J. Pou, J. Zaragoza, G. Konstantinou, and V. G. Agelidis, "Closed-loop discontinuous modulation technique for capacitor voltage ripples and switching losses reduction in modular multilevel converters," IEEE Transactions on Power Electronics, vol. 30 , no. 9, pp. 4714-4725, Sep. 2015.

[39] P. Ghimire, A. R. de Vega, S. Beczkowski, B. Rannestad, S. Munk-Nielsen, and P. Thogersen, "Improving power converter reliability: Online monitoring of high-power igbt modules," IEEE Industrial Electronics Magazine, vol. 8, no. 3, pp. 40-50, Sep. 2014.

[40] Z. Li, J. Guo, and R. Zhou, "Maintenance scheduling optimization based on reliability and prognostics information," in 2016 Annual Reliability and Maintainability Symposium (RAMS), Jan 2016, pp. 1-5.

[41] M. Andresen, V. Raveendran, G. Buticchi, and M. Liserre, "Lifetime-based power routing in parallel converters for smart transformer application," IEEE Transactions on Industrial Electronics, vol. PP, no. 99, pp. 1-1, 2017.

[42] V. Raveendran, M. Andresen, and M. Liserre, "Improving onboard converter reliability for more electric aircraft with lifetime-based control," IEEE Transactions on Industrial Electronics, vol. 66, no. 7, pp. 5787-5796, July 2019.

[43] S. Pelley, D. Meisner, P. Zandevakili, T. F. Wenisch, and J. Underwood, "Power routing: dynamic power provisioning in the data center," in ASPLOS, 2010

[44] M. Andresen, V. Raveendran, G. Buticchi, and M. Liserre, "Lifetime-based power routing in parallel converters for smart transformer application,” IEEE Transactions on Industrial Electronics, vol. 65, no. 2, pp. 1675-1684, Feb 2018.

[45] Y. Ko, V. Raveendran, M. Andresen, and M. G. Liserre, "Thermally-compensated discontinuous modulation for mvac/lvdc building blocks of modular smart transformers," IEEE Transactions on Power Electronics, pp. 1-1, 2019.

[46] 1200 V three phase PIM IGBT module, Infineon Technologies AG, 10 2013, rev. 2.0.

[47] M. Musallam and C. M. Johnson, "An efficient implementation of the rainflow counting algorithm for life consumption estimation," IEEE Transactions on Reliability, vol. 61, no. 4, pp. 978-986, Dec 2012.

[48] D. Zhou, H. Wang, F. Blaabjerg, S. K. Koer, and D. Blom-Hansen, "System-level reliability assessment of power stage in fuel cell application," in 2016 IEEE Energy Conversion Congress and Exposition (ECCE), Sept 2016, pp. 1-8.

[49] V. Raveendran, M. Andresen, and M. Liserre, "Lifetime control of modular smart transformers considering the maintenance schedule," in 2018 IEEE Energy Conversion Congress and Exposition (ECCE), Sep. 2018, pp. 60-66.

[50] A. Marquez, J. I. Leon, S. Vazquez, and L. G. Franquelo, "Closed-loop active thermal control via power routing of parallel dc-dc converters," in 2018 IEEE 12th International Conference on Compatibility, Power Electronics and Power Engineering (CPE-POWERENG 2018), April 2018, pp. 1-6.

[51] A. Marquez, J. I. Leon, S. Vazquez, L. G. Franquelo, G. Buticchi, and M. Liserre, "Power device lifetime extension of dc-dc interleaved converters via power routing," in IECON 2018 - 44th Annual Conference of the IEEE Industrial Electronics Society, Oct 2018, pp. $5332-5337$.

[52] J. I. Leon, S. Kouro, L. G. Franquelo, J. Rodriguez, and B. Wu, "The essential role and the continuous evolution of modulation techniques for voltage-source inverters in the past, present, and future power electronics," IEEE Transactions on Industrial Electronics, vol. 63, no. 5, pp. 2688-2701, May 2016.

[53] J. I. Leon, S. Vazquez, and L. G. Franquelo, "Multilevel converters: Control and modulation techniques for their operation and industrial applications," Proceedings of the IEEE, vol. 105, no. 11, pp. 2066-2081, Nov 2017.

[54] Y. Ko, M. Andresen, G. Buticchi, and M. Liserre, "Power routing for cascaded h-bridge converters," IEEE Transactions on Power Electronics, vol. 32, no. 12, pp. 9435-9446, Dec 2017.

[55] Y. Ko, V. Raveendran, M. Andresen, and M. G. Liserre, "Advanced discontinuous modulation for thermally compensated modular smart transformers," IEEE Transactions on Power Electronics, pp. 1-1, 2019.

[56] V. G. Monopoli, A. Marquez, J. I. Leon, Y. Ko, G. Buticchi, and M. Liserre, "Improved harmonic performance of cascaded h-bridge converters with thermal control," IEEE Transactions on Industrial Electronics, vol. 66, no. 7, pp. 4982-4991, July 2019.

[57] A. Marquez, J. I. Leon, S. Vazquez, R. Portillo, L. G. Franquelo, E. Freire, and S. Kouro, "Variable-angle phase-shifted pwm for multilevel three-cell cascaded h-bridge converters," IEEE Transactions on Industrial Electronics, vol. 64, no. 5, pp. 3619-3628, May 2017.

[58] A. Marquez Alcaide, V. G. Monopoli, J. I. Leon, Y. Ko, G. Buticchi, S. Vazquez, M. Liserre, and L. G. Franquelo, "Sampling-time harmonic control for cascaded h-bridge converters with thermal control," IEEE Transactions on Industrial Electronics, vol. 68, no. 4, pp. $1-1,2020$.

[59] A. Marquez Alcaide, J. I. Leon, V. G. Monopoli, S. Vazquez, M. Liserre, and L. G. Franquelo, "Generalized harmonic control for chb converters with unbalanced cells operation," IEEE Transactions on Industrial Electronics, in press. 
[60] N. Ghorbani and E. Babaei, "Exchange market algorithm," Applied Soft Computing, vol. 19, pp. 177 - 187, 2014. [Online]. Available: http://www.sciencedirect.com/science/article/pii/S156849461400074X

[61] X.-J. Cai, Z.-X. Wu, Q.-F. Li, and S.-X. Wang, "Phase-shifted carrier pulse width modulation based on particle swarm optimization for cascaded h-bridge multilevel inverters with unequal dc voltages," Energies, vol. 8, no. 9, pp. 9670-9687, 2015. [Online]. Available: https://www.mdpi.com/1996-1073/8/9/9670

[62] M. Liserre, A. Marquez, M. Andresen, J. I. Leon, S. Vazquez, Y. Ko, and L. G. Franquelo, "Method for converting electrical power, computer program and electrical power converter,” European Patent EP19180419.4, 2019. 